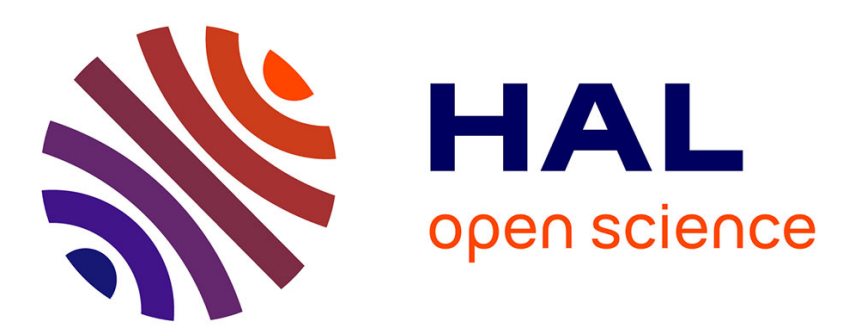

\title{
L'UTILISATION DES FAISCEAUX D'IONS LOURDS, "NOUVELLE" ou "VIEILLE" PHYSIQUE NUCLEAIRE?
}

\author{
M. Lefort
}

\section{- To cite this version:}

M. Lefort. L'UTILISATION DES FAISCEAUX D'IONS LOURDS, "NOUVELLE" ou "VIEILLE" PHYSIQUE NUCLEAIRE?. Congres General de la Societe Francaise de Physique, 1979, Toulouse, France. pp.C3-65-C3-76, 10.1051/jphyscol:1980311 . jpa-00219830

\section{HAL Id: jpa-00219830 https://hal.science/jpa-00219830}

Submitted on 1 Jan 1980

HAL is a multi-disciplinary open access archive for the deposit and dissemination of scientific research documents, whether they are published or not. The documents may come from teaching and research institutions in France or abroad, or from public or private research centers.
L'archive ouverte pluridisciplinaire HAL, est destinée au dépôt et à la diffusion de documents scientifiques de niveau recherche, publiés ou non, émanant des établissements d'enseignement et de recherche français ou étrangers, des laboratoires publics ou privés. 


\title{
L'utilisation des faisceaux d'ions lourds, "Nouvelle" ou "Vieille" Physinue NUCLEAIRE ?
}

\author{
M. Lefort \\ I. P. N. Orsay et Ganil caen.
}

\begin{abstract}
Résumé : Quelques aspects nouveaux et originaux apportés à la physique nucléaire par les ions lourds sont présentês. Du point de vue des techniques expérimentales, ce sont les faisceaux, les méthodes d'identification de masse et de $Z$, les coincidences multiples et les collections de noyaux. Du point de vue de la connaissance en physique nucléaire, c'est une comprëhension d'un micro-ensemble de matiēre condensée. On décrit les grandes lignes des réactions qualifiées de "dissipatives" et les aspects de mécanique statistique appliquée au fluide nuclēaire, ainsi que l'estimation de la durée des phénomènes. On montre ensuite comment les idées de base "anciennes" de la physique du début du sièce sont fertiles pour décrire macroscopiquement les noyaux.
\end{abstract}

Abstract : Heavy ions have brought to nuclear physics a new spirit, both for instrumental developments and for the basic concept of nuclear matter as condensed phase. Dissipative reactions are described in terms of statistical mechanics and transport equations. Also time scales are given. It is shown how fertile is the "old" basic background of physics for a macroscopic description of nuclei.

Je voudrais, à ce congrès 1979 de la Société de Physique, évoquer quelques idées générales sur l'évolution de la physique nucléaire depuis une dizaine d'années, et sur le renouveau que les ions lourds ont apportê aussi bien dans les méthodes expérimentales que dans les concepts de la connaissance du noyau et de 1 a matière nucléaire. Ainsi, le titre sacrifie-t-il à la mode. Pour justifier l'intérêt d'un sujet, d'un domaine, d'un produit, il doit être nouveau : les nouveaux philosophes, la nouvelle cuisine, le nouveau jazz,..., tout en mettant en relief l'attrait des coutumes, des idées et des choses du début du 19e siècle. Nous n'échappons pas à ces "humeurs". Ainsi, Roger Caillois ${ }^{1}$ écrivait-il, quelque temps avant sa mort en fin de 1'année 1978 : "Je me suis aperçu très tôt que 1a science était trop changeante, qu'une découverte $y$ chassait l'autre et qu'il n'était en elle de stable que l'intention qui la soutenait. " C'est probablement cette intention d'une grande constance qui conduit les physiciens nucléaires depuis 40 ans à proposer, à rêclamer, quelquefois à obtenir la construction et la mise en marche de nouveaux accêlérateurs. Il est vrai en tout cas, que, depuis l'époque de la découverte des constituants du noyau le modèle en association de particules $\alpha$ a été chassé par la goutte liquide elle-même remise dans l'armoire des souvenirs par le modēle en couche à particules indēpendantes jusqu'à ce que les partisans les plus convaincus de l'étude systématique. des états individuels de nucléons finissent au cours des années 70 par se précipiter vers la "macrophysique" nucléaire ou "chimie nucléaire", étude de processus qu'ils considéraient autrefois comme si complexes qu'aucune information intēressante et aucune compréhension ne pourraient en être tirées.

En réalité, grâce aux faisceaux d'ions lourds, d'importantes limitations imposées par 60 ans de physique nucléaire ont été levées, et de nombreuses idées appliquées dans les autres domaines de physique depuis la fin du 19e siècle sont venues fertiliser les champs de la connaissance du milieu de matière très condensée qu'est le noyau. Ces limitations étaient principalement que seules les formes sphériques ou presque sphériques de noyaux êtaient connues et accessibles, que le nombre de $Z$ et de $A$ était restreint, que les noyaux étajent gēnéralement à 1 'ētat fondamental ou à des états excités correspondant à des températures et à des pressions que l'on peut qualifier de normales. Essentiellement, deux types de mécanismes de réactions initiées par des faisceaux. de nuclēons étaient connus, celui du noyau composé, depuis Bohr, Weisskopf, Feschbach, et celui dit des réactions directes au cours desquelles le 
proton ou le neutron incident interagissait idéalement avec un seur nucléon du noyau cible. Enfin et surtout toutes les idées avaient pour point de départ le modēle atomique, c'est-à-dire une description totalement régie par la mécanique quantique des mouvements individuels de nucléons par des fonctions d'onde dans un puits de potentiel. Et toutes les propositions d'expériences avaient pour objectif la mise en évidence de la structure des noyaux par des réactions nucléaires les plus "êlémentaires" possible au point de vue du mécanisme. Les dênominations les plus répandues illustrent cet état d'esprit : modèle optique, méthode DWBA (Approximation de Born d'onde distordue), oscillateur harmonique, excitation trou-particule, etc... Les aspects collectifs et surtout la notion de fluide nucléaire n'apparaissaient qu'occasionnel lement (résonnante géante, fission) et leur étude fondamentale était souvent laissêe de côté et considérée comme sans grand intêrêt pour la connaissance de la structure des noyaux, dès lors qu'une forme et une profondeur de potentiel étaient choisies.

\section{Pourquoi "nouvelle" physique nuclēaire ?}

11 nous faut parler d'abord des nouveautés de caractère technique grâce auxquelles les informations recueillies au cours des expériences sont plus nombreuses, plus précises et cernent mieux les réponses aux questions.

Les faisceaux d'ions lourds, en 1960 curiosités pour quelque ions, ${ }^{12} \mathrm{C},{ }^{16} 0,{ }^{20} \mathrm{Ne}$, sont maintenant accēlérés dans presque tous les centres de recherches nucléaires, soit à la suite d'adaptation des cyclotrons des linéaires et des électrostatiques existants, soit grâce à la construction de nouvelles machines conçues pour fournir à des ions de masses de plus en plus lourdes des énergies supérieures aux barrières coulombiennes. Les progrès des sources d'ions classiques (sources Pig) ont permis de produire des ions suffisamment multichargés pour que le rapport $q / m$ de charge à masse atteigne des valeurs permettant l'accélération dans les cavités résonnantes., Cependant, ce rapport restait encore insuffisant pour que des énergies. élevées soient atteintes. L'épluchage des ions ayant déjà une certaine vitesse et traversant un "rideau" de matière de quelques dizaines de microgrammes par $\mathrm{cm}^{2}$ a conduit au système du double accélérateur, avec une section basse énergie por- tant les ions à des vitesses approchant $\beta=0,05$, et une section haute énergie qui suit 1'éplucheur grâce auquel le rapport $q / m$ est accru d'un facteur 3 a 5 selon les valeurs de $\beta$, ce qui permet un deuxième étage très efficace d'accélération. ${ }^{2}$

Ajoutons que les développements trẽs récents de nouvelles sources inspirées de la physique des plasmas permet d'envisager dans un futur pas trop lointain, d'atteindre avec un seul accélérateur les performances en énergie des deux étages dans la mesure où ces sources fournissent un taux d'épluchage très élevé avec une intensité raisonnable (Sources Cryebis ${ }^{3}$, Sources ESR) 4 .

Dans l'état actuel des machines, 11 est donc possible, par exemple dans le double accélérateur linéaire de Darmstadt (G.S.I.) de précipiter $10^{11}$ noyaux d'uranium sur une cible d'uranium avec une énergie de $2,5 \mathrm{GeV}$ (10 MeV par nucléon).

Examinons maintenant les moyens de dētection et les informations recueillies dans les expériences. Lorsque les projectiles étaient des protons ou des particules $\alpha$, le résultat de la réaction était surtout un noyau résiduel accompagné d'un ou quelques nucléons et de rayonnement $\gamma$. Le principal objectif était de mesurer avec précision l'énergie de ces particules et leur distribution angulaire. Les problèmes d'identification des produits étaient relativement simples. A partir du moment où le projectile est lui-même un noyau complexe, oũ les voies de sorties peuvent être très variées, deux difficultés expérimentales supplëmentaires sont à résoudre.

a) Identifier le mieux possible les masses et les charges des produits, fragments projetés divers de toutes masses et charges depuis celle des protons jusqu'à celle du noyau composé.Ainsi, d'énormes progrès ont été faits pour l'identification de masse, grâce au gain de précision des temps de vol, donc aux performances de l'électronique rapide. Le signal de passage d'une particule dans un détecteur est maintenant couramment dêterminé grâce aux galettes à microcanaux, avec une incertitude en temps de 150 picosecondes de sorte que sur une base de temps de seulement $100 \mathrm{~cm}$, on peut obtenir $\Delta t / t=5.10^{-3}$ pour une particule de plusieurs $\mathrm{MeV}$ par nucléon $(\beta=0,1)$. L'ēnergie étant mesurēe avec une résolution de $10^{-2}$, on peut donc obtenir une résolution en masse $\Delta m / m=2 \Delta t / t+\Delta E / E$ de $2 / 100$, autrement dit séparer les masses jusqu'à 
$\left.A=50 . L^{\prime} u t i\right] i s a t i o n$ de spectromètres magnétiques permet d'aller plus loin grâce à l'information de rigiditê magnétique proportionnelle à la quantité de mouvement. De même, les progrès des détecteurs de mesure de perte d'énergie $\Delta E$, surtout concernant 1 'homogénēité et l'uniformité de 1 'épaisseur ont permis 1 'identification en $Z$ jusqu'à $Z=40$ par mesures du produit $\triangle E \times E$, proportionnel à $M z$ selon la loi de Bethe, $z$ est la charge de I'ion, directement liée au numéro atomique lorsque l'énergie est connue (voir la figure 1).

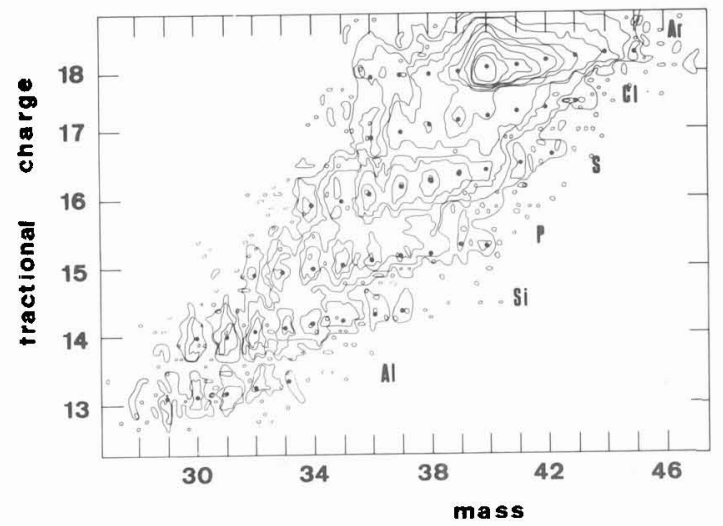

Fig.1. Courbes de niveau obtenues pour les nombres d'evenements correspondant cux différentes masses des produits de rëaction $\left({ }^{40} A r+{ }^{238} U\right) \cdot$ Réf. $^{5}$.

b) Pour comprendre ce qui s'est passé au cours de la collision, la mesure d'un seul des produits de réaction $n$ "est plus une information suffisante.Dans les cas les plus simples, deux fragments sont émis. La connaissance de la corrêlation angulaire et du bilan énergêtique (cinématique) est essentielle. Le rôle des mesures en coïncidences est donc primordial. Dans un premier stade, les expériences inclusives fournissent des données d'ensemble, mais très vite des expériences exclusives sont nécessaires. Pour les ions lourds de très grande énergie, le nombre de détecteurs opérant en coīncidence devient grand. La complexité de la logique d'analyse des résultats croît. On se rapproche des méthodes de la physique des particules. Il suffit de regarder la photographie des traces ${ }^{6}$ de la figure 2 qui ressemble à une explosion d'étoile pour se convaincre des problèmes expérimentaux à résoudre (Argon sur Plomb à $1,8 \mathrm{GeV} / \mathrm{n}$ )

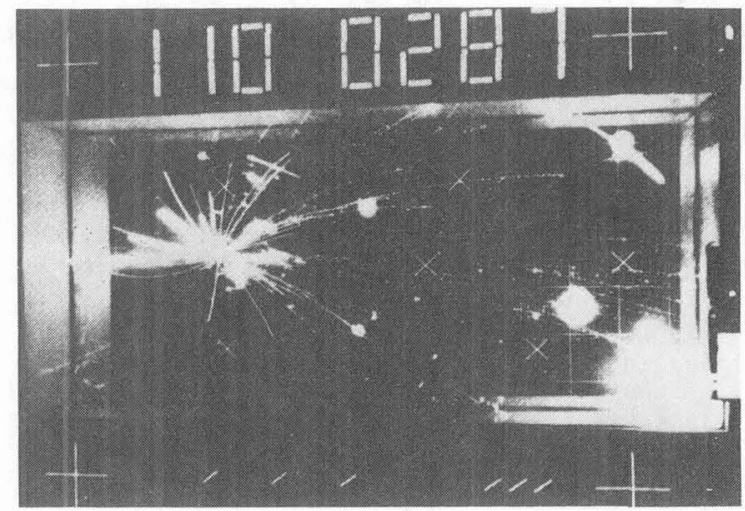

Fig. 2. Photographie d'une explosion de noyau dans la collision centrale $(A r+P b)$ à une énergie de 1,8 GeV par nucléon (Cliché de Schroeder. Réf. ${ }^{6}$.)

De nouveaux systèmes de détection sont apparus, permettant l'acquisition non seulement de l'énergie totale mais de la localisation dans l'espace et des pertes d'ênergie le long de la trajectoire ${ }^{7}$ (chambres à fils, chambres à plaques parallèles, etc...).

c) Un autre aspect expêrimental est la grande variété des espẽces nucléaires synthétisêes par les réactions entre noyaux. Après la collision, les noyaux de recul "exotiques", c'est-à-dire de rapports neutron-proton très loin des conditions de stabilité, doivent être recueillis rapidement, transportés loin du faisceau et leur radioactivité doit être êtudiēe dans un délai très court car leur grande instabilité entraine une faible durée de vie. On a inventé de nouveaux procédés, soit dans les sources $d^{\prime}$ ions des séparateurs $d^{\prime}$ isotope en ligne, soit sur des systèmes de transport par jets gazeux. La 
"nouvelle" physique s'est souvenue des acquis de la "vieille" chimie, pour les propriétés de diffusion des élëments dans les collecteurs de graphite, ou pour la formation d'aérosols, ã partir d'impuretés organiques ionisées par le faisceau traversant de 1 'hêlium. Ces aérosols chargês fixent les noyaux projetēs hors de la cible et sont entrainés avec l'hétium pompé à travers un capillaire. En moins de quelques millisecondes, les noyaux radioactifs sont emportês par leur convoyeur à plusieurs mètres et l'impact des aérosols massifs-arrivant avec la vitesse du son dans l'hélium sur le collecteur à la sortie du capillaire fixe les noyaux sur ce collecteur. A partir de là, des dispositifs ingênieux ont été montés ${ }^{8}$ permettant, outre la mesure de la radioactivité $\alpha$ ou $\beta$ et des rayonnements $\gamma$, ]'identification en masse par temps de vol du noyau émetteur de rayonnement et reculant dans un dispositif spécial (voir photo figure 3 ).

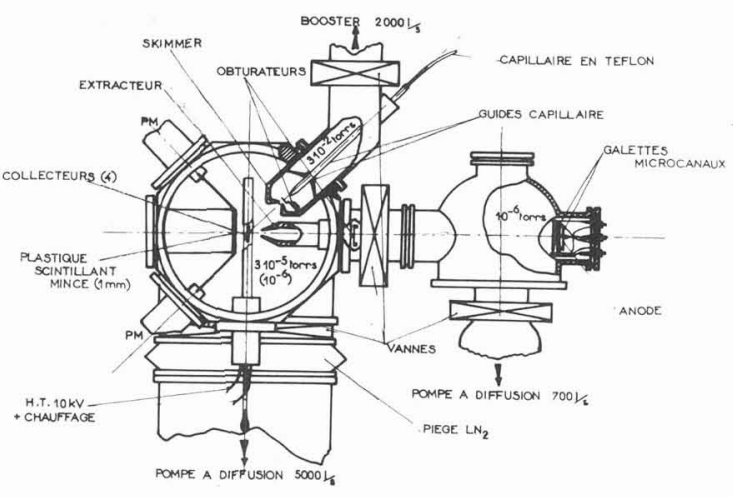

Fig.3. Schéma d'une installation recueillant les noyaux produits à une distance de 7 mètres et transportés par jet d'hélium vers un dispositif permettant la mesure de la radioactivité et la détermination de la masse des noyaux reculant (Accélérateur ALICE d̀ Orsay. Réf. ${ }^{8}$ ).

Je $n^{\prime}$ à cité que quelques exemples, mais il suffit de passer dans une salle d'expériences auprès d'un accélérateur d'ions lourds pour observer combien les dispositifs ont changé en dix ans et surtout combien les informations sortant des périphériques des ordinateurs ont évolué dans leur présentation aussi bien que dans leur signification profonde (courbes de niveau, tableaux multiparamétriques, etc...)

Passons maintenant au sujet d'étude lui-même. La caractéristique principale de la nouvelle approche de notre connaissance du noyau réside dans un essai de compréhension de celui-ci en tant qu'un micro-ensemble de matière condensée. En effet, que se passe-t-il lorsqu'un noyau, ensemble compact de $A_{1}$ nucléons rencontre avec suffisamment d'énergie un autre noyau de $A_{2}$ nucléons ? Le schēma de trajectoires de la figure 4 indique les diverses possibilités selon les paramètres d'impact et selon les fonctions de déflexion $\theta=f(b)$. Dans le cas

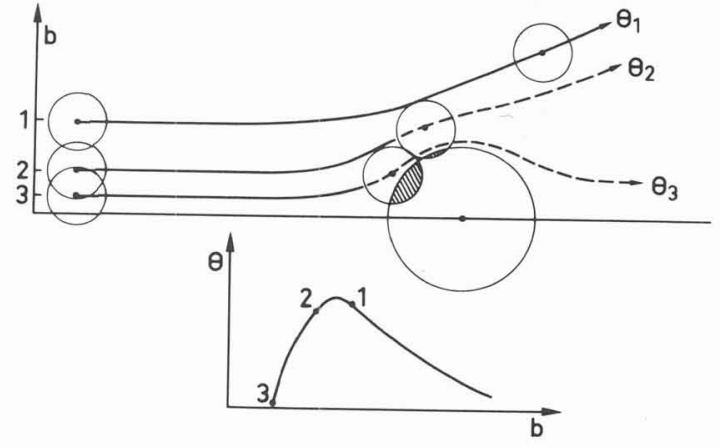

Eig.4. Schéma des diverses possibilités de. trajectoires entre un ion lourd et un noyau cible. Les numéros correspondent aux explications du texte. La trajectoire frontale conauisant à la fusion complète n'est pas figurée. La fonction de déflexion est indiquée.

$n^{\circ} 1$, seule $1^{\prime}$ interaction coulombienne intervient. Mais le fait que le produit des charges soit êlevê, crée des effets d'excitation des niveaux d'ênergie des nucléons dans le noyau inaccessibles avec des projectiles légers. Déjà pour ces interactions, un aspect nouveau de ce qu'on appelle l'excitation 
coulombienne apparaît. Sur des noyaux très lourds, elle peut être suffisante pour provoquer une oscillation de la matière nucléaire conduisant à la fission. Telle est la découverte toute récente (Juin 1979) de Specht et ses collaborateurs à Darmstadt.9 L'histoire de cette découverte est édifiante. Comment en effet prouver que la fission de l'uranium observée par la détection de deux fragments en coīncidence est bien provoquée par un pur effet coulombien sans intervention de réaction nucléaire ? D'abord, l'expérience est effectuée à uné énergie dite sous le seuil de Coulomb $E<\frac{Z_{1} Z_{2} e^{2}}{R_{1}+R_{2}}$, mais $l$ 'on sait que des transferts de neutrons peuvent avoir lieu, même dans ces conditions énergétiques. Il faut donc repérer le projectile qui repart après diffusion Rutherford. Ce projectile, dans le cas présent ${ }^{184} \mathrm{~W}$, doit avoir été excité seulement par l'interaction électromagnètique. $0 r$, on sait par ailleurs qu'un état excité de ${ }^{184} \mathrm{~W}$ se désexcite par conversion interne d'électrons avec une forte probabilitê et avec une durêe de vie connue. L'idée remarquable de Specht est donc de détecter cet électron de conversion émis lorsque le projectile est déjà revenu en arrière à une distance correspondant à la durée de vie du niveau excité, en coīncidence avec les deux fragments. Ainsi, la nouvelle physique celle de l'oscillation collective de ce fluide nucléaire oscillant jusqu'à fissionner, et l'ancienne physique, celle des niveaux bien repérês et nomenclaturés du modèle à particules indépendantes se marient-elles pour observer ce comportement collectíf de la matière nucléaire instable.

Continuons avec la trajectoire $n^{\circ} 2$, où ies noyaux se frôlent. C'est le domaine des transferts de nucléons qui a eu son apogée pendant une dizaine $d^{\prime}$ années. Interférences des fonctions d'onde autour de l'angle d'arc-en-ciel, oscillations dans les distributions angulaires, sélectivité des caractéristiques quantiques des nuclēons échangēs, pendant quelques années, la"vraie" physique méprisait les interactions profondes et la "cuisine" de la fusion de deux noyaux entre eux.

La 3e trajectoire n'existait pas avant 1969. Elle ne pouvait exister. Ou bien l'interaction rasante est directe, avec légère perturbation nucléaire de l'interaction coulombienne et échange d'un nucléon, ou bien le projectile est absorbé par la cible parce que pénétrant selon une trajectoire trop frontale et 1 'ensemble des nucléons $A_{1}+A_{2}$ est le noyau composé deNiels Bohr. Et pourtant, une situation intermédiaire est apparue, qui peut se résumer schëmatiquement ains $i^{10}:$ lorsque les forces nucléaires attractives dues à l'interpénétration des deux ensembles sont suffisantes par rapport aux forces coulombiennes et centrifuges, un "accrochage" de ces noyaux a lieu. Le système, encore déformé tourne avec une vitesse d'autant plus grande que le choc est moins frontal (voir Fig.5)

$$
\omega=\frac{d \theta}{d t}=\frac{l \hbar}{y}
$$

oũ le moment d'inertie $J$ dépend de la déformation. On utilise souvent le modèle de deux sphères reliēes par un col. L'énergie cinétique initiale peut alors être totalement transformée en énergie interne, puis dans un stade ultime, la reséparation en deux fragments a lieu, mais l'énergie cinétique de ces deux morceaux est entièrement attribuable à la seule rêpulsion des charges, comme dans la fission nucléaire. Dans un stade ultérieur, Tes deux fragments libèrent l'énergie d'excitation due à la perte d'énergie cinétique initiale et au $Q$ de la réaction sous forme d'émission de nucléons, de particules $\alpha$ et de rayonnement. Le temps est cependant suffisant pour que de nombreux échanges de nucléons aient lieu à travers le col et pour que des vibrations collectives de 1 'ensemble produisent des effets. Le résultat final est qu'au lieu de trouver deux fragments très voisins des partenaires de la voie d'entrëe, on observe toute une distribution de masses et de numëros atomiques. L'accumulation des données qui ont permis de décrire ainsi le phēnomène est maintenant impressionnante. Lorsque les projectiles et les cibles dépassent les masses moyennes $(A=180)$, presque toutes les ondes partielles conduisent à ces phénomènes, car même les chocs frontaux ne peuvent plus conduire à la fusion complète.

Enfin, lorsque l'ênergie croît, le choc de plus en plus brutal conduit à des projections immédiates de quelques nucléons avant ou pendant 1 'accrochage. La température interne du système monte. A très haute énergie, lorsque les vitesses deviennent une fraction importante de la vitesse de la lumière (GeV par nucléon), les chocs très frontaux provoquent un véritable éclatement des noyaux en dizaines de morceaux et nucléons, tandis que pour les chocs périphériques, le projectile traversant le bord de la cible est "chauffé" en une boule de feu. ${ }^{2} \mathrm{La}$ transition entre les énergies de $10 \mathrm{MeV}$ par 


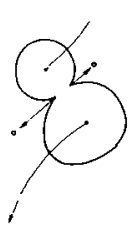

(1)
(2)

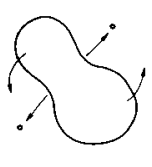

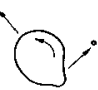<smiles>CC1CCCCC(C)C1</smiles>

$v_{k} v_{0}$

$(3)$
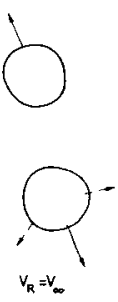

(4)
Fig.5. Schéma d'une interaction dissipative entre deux noyaux. L'émission de particules légères peut avoir lieu à différentes étapes.

nucléon oũ la température est encore en dessous de l'ênergie de liajson des nuclêons, et les très hautes ënergies, est presque inconnue. Elle correspond au domaine de 20 à $100 \mathrm{MeV}$ par nucléon pour lequel i) n'existe actuellement aucun accélérateur.

Or, cette région d'énergie indiquée sur $1 \mathrm{a}$ figure 6 est cruciale. En effet, la distribution de Fermi des nucléons dans un noyau est répartie autour d'une vitesse de $\beta=0,2$ soit $20 \mathrm{MeV} /$ nucléon, de sorte que le mouvement d'approche sera comparable aux mouvements internes pour cette énergie cinêtique. Au delà de 100 ou $200 \mathrm{MeV}$ par nucléon, $1 \mathrm{a}$ longueur d'onde associēe à chaque nucléon devient très infërieure aux distances d'interaction entre nucléons dans le noyau et le projectile ne se comporte plus comme un amas cohérent. La température moyenne cédêe au cours d'une collision ne peut guère dépasser $8 \mathrm{MeV}$, ënergie de liaison des nuclēons puisqu'au delà c'est l'évaporation immédiate. Entre $10 \mathrm{MeV}$ par nucléon et $100 \mathrm{MeV}$ par nucléon, on chauffe les noyaux entre 2 et $8 \mathrm{MeV}$ et cette zone de noyaux très chauds présente un grand intérêt.

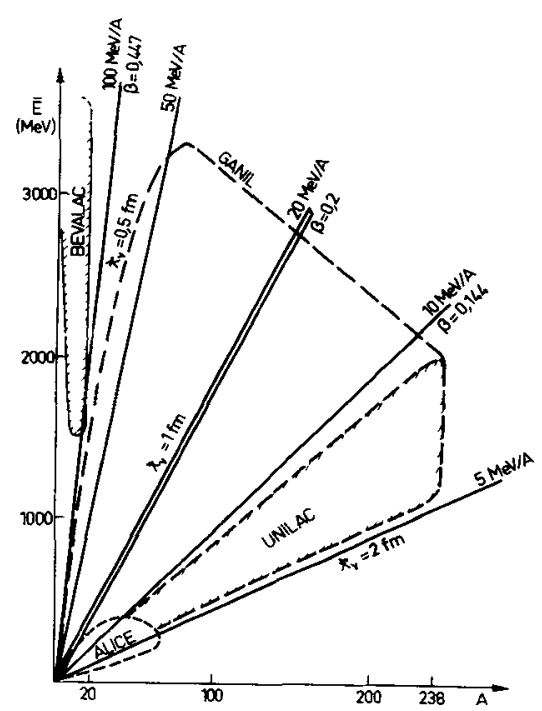

Eig.6. Présentation des vitesses et énergies des ions zourds en fonction de Zeur masse. Les vitesses sont indiquées par rapport $\grave{a}$ Ia vitesse de Za Zumière, $\beta$. La Zongueur d'onde associée d̀ chaque nucléon incident est $k$. Les domaines des accélérateurs ALICE (Orsay), UNILAC (Darmstadt), BEVALAC (Berkeley) et GANIL (Caen) sont figurés.

Revenons donc à cette énorme perte d'énergie cinêtique observée lorsque deux noyaux se rencontrent, perte mettant en évidence une viscosité nuclēaire insoupçonnée et des effets de friction rappelant la physique des fluides incompressibles. Peut-on comprendre ces phēnomènes dissipatifs du point de vue microscopique ? Une analyse phënomënologique quantitative en a été faite grâce aux concepts de friction, de viscosité dëfinis en dynamique des fluides et en mécanique statistique. Les paramètres utilisés peuvent-ils être calculés sur la base des propriêtēs des noyaux?

Les processus irréversibles observés dans ces réactions dissipatives sont le résultat d'un nombre d'interactions trop grand pour un calcul complet de type microscopique (couplage avec les êtats de particules individuelles), et trop petit pour que les arguments habituels de mécanique statistique soient valides sans un examen critique. En effet, le nombre de nucléons interagissant ne dêpasse guère 100. Il s'agit là d'une situation unique d'ensembles à mi-distance de la microphysique et de la macrophysique. 
Examinons aussi les échelles de temps. A $10 \mathrm{MeV}$ par nucléon, la durée d'une collision élastique ou d'une interaction directe n'est que de quelques $10^{-23} \mathrm{sec}$. Nous prendrons cette unité qui peut être associêe à une longueur d'onde d'environ $\lambda=0,1 \mathrm{fm}$ pour une énergie de $50 \mathrm{MeV}$ au dessus de la barrière coulombienne et pour une masse $A=50$. La durée d'une rotation d'un noyau de masse moyenne disposant d'un moment angulaire d'environ $50 \mathrm{l} \mathrm{l}^{2}$ peut être calculée en prenant le moment d'inertie d'une sphère rigide :

$$
\tau_{R}=\left(\frac{\theta}{\omega}\right)_{2 \pi}=\frac{2 \pi}{\ell \hbar} \# 10^{-20} \text { sec }=10^{3} \text { unités }
$$

La durée de vie d'un noyau composē peut être estimée. La largeur d'émission de particules par évaporation est approximativement

$$
\Gamma=14 \exp \left(-4,7 \sqrt{A / E^{*}}\right)
$$

et la relation $\Gamma \tau_{N C}=\hbar$ donne par exemple pour une énergie d'excitation de $300 \mathrm{MeV}$ dans un noyau $A=100$, une durée de vie de $10^{-21} \mathrm{sec}$, plus courte que 1 a durée de rotation. Cependant pour les énergies d'excitation plus généralement atteintes de 50 à $100 \mathrm{MeV}, \tau_{N C}$ est entre $10^{-19}$ et $10^{-20} \mathrm{sec}$. Le temps de relaxation nécessaire pour dissiper l'énergie initiale peut être estimé de différentes façons, et aussi mesuré expérimentalement. Par exemple, on peut observer dans une distribution angulaire des fragments (voir figure 7), à quel angle $\theta$ la relaxation totale de l'énergie cinétique est achevée, et si $\theta_{r}$ est l'angle d'effleurement rasant ${ }^{13}$

$$
\theta_{r}-\theta=\langle\omega\rangle=\frac{\ell_{f} \hbar}{g}
$$

où $\langle\omega\rangle$, la vitesse angulaire moyenne du système est donnée par le moment angulaire orbital en voie de sortie

$$
\ell_{f}=\ell_{i}-\left(J_{1}+J_{2}\right)
$$

où $\mathrm{J}_{1}$ et $\mathrm{J}_{2}$ sont les moments angulaires propres acquis par chaque fragment, et est le moment d'inertie du système tournant. Si le système est totalement "accroche"

$$
\begin{aligned}
& e_{i} \hbar=\omega\left(y_{1} y_{1}+y_{2}\right) \\
& \text { avec } \quad y_{1,2}=\frac{2}{5} A_{1,2} R_{1,2}^{2}
\end{aligned}
$$

le temps de rotation ainsi estimé est de quelque $10^{-21} \mathrm{sec}$. Il est donc intermédiaire entre la durée de vie d'un noyau composé et la durêe des interactions individuelles des nucléons dans un noyau (quelque $10^{-23}$ secondes).

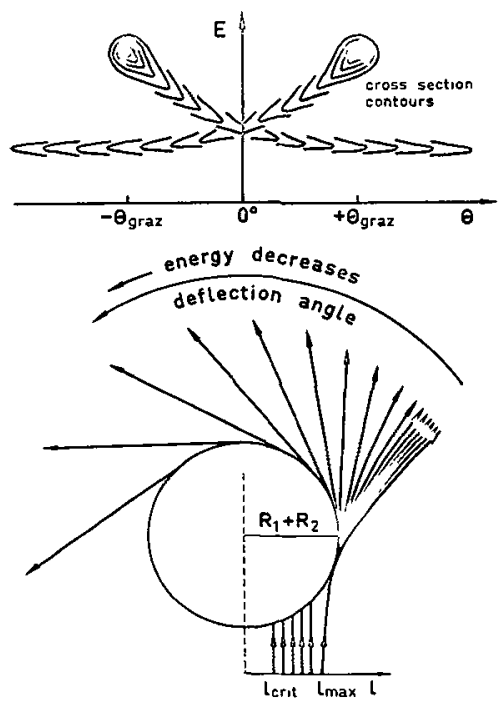

Fig.7. Schéma de principe des courbes de niveau correspondant aux sections efficaces différentielles $\frac{d 2 \sigma}{d \theta a}$, et dessin des trajectoires.

Ce dessin visualise la contribution des évènements issus des angles dits "négatifs" pour tout détecteur placé à un angle $\theta$ donné. (Réf. ${ }^{13}$ )

Par exemple, la durêe associée à la création d'une paire trou-particule par un seul transfert de quantîté de mouvement $\hbar \Delta k$, peut être estimée, sachant que le moment de Fermi est $\hbar \mathrm{k}_{F}=1,36 \hbar \mathrm{fm}^{-1}$ et qu'en surface, la densité nucléaire étant plus faible, on doit prendre une valeur plus faible. On arrive ainsi à une durée de 1 'ordre de $10^{-22} \mathrm{sec}$. Quant à la durée de vie d'un état de $\mathrm{N}$ trousparticules de largeur typique $\Gamma=200 \mathrm{keV}$, elle est donnée par $\theta=\frac{\hbar}{4 N . \bar{T}}$ soit environ $5.10^{-23} \mathrm{sec}$ pour une vingtaine de paires trous-particules.

Avec ces nombres en tête, on peut estimer la durée de la phase d'approche entre deux noyaux. Au temps $t=0$, la première paire trou-particule est formée et à $\tau=4 \cdot 10^{-23} \mathrm{sec}$ la deuxième. Chaque état individuel décroit avec $\theta=\hbar / 2.200 \mathrm{keV}=16.10^{-2} 2_{\mathrm{sec}}$ La probabilité $P_{n}$ de trouver après $n$ transferts de moment les $n$ paires originellement créées non perturbées est

$$
P_{n}=\exp \left(-n(n+1) \frac{\tau}{\theta}\right)
$$

On obtient 1 e après 6 collisions soit $t=n \tau=2,5 \cdot 10^{-22} \mathrm{sec}$. 
Si le transfert d'énergie à chaque $\Delta k$ est $5 \mathrm{MeV}$, cela correspond à $30 \mathrm{MeV}$. Cette phase d'approche ne peut être traitée de façon statistique.

Par contre, on peut admettre qu'ensuite, un bain thermique s'est créé entre les états d'énergie intrinsèques et que les idées générales des théories de transport et de diffusion vont pouvoir s'appliquer. Les principales variables collectives examinées sont le rapport neutron proton (N/Z) liē à l'isospin et fondamental dans la stabilité des noyaux, le mouvement relatif des deux ensembles (perte d'énergie cinétique) décomposē en radial et tangentiel (perte de moment angulaire orbital), l'asymétrie de masse (transfert de nucléons) et la déformation. On a maintenant une idée des durées d'équilibration de ces degrés de libertê, indiquées dans le tableau suivant en unités $10^{-23} \mathrm{sec}$.

\begin{tabular}{|c|c|c|c|c|c|}
\hline${ }^{\top} N / Z$ & ${ }^{\top} \varepsilon_{\text {rad }}$ & ${ }^{\top} \varepsilon_{\text {rot }}$ & ${ }^{\tau}{ }_{m}$ & ${ }^{\tau}$ def & ${ }^{\tau}$ \\
\hline 10 & 50 & 100 & 500 & 500 & $\sim 500$ \\
\hline
\end{tabular}

Puisqu'un bain à température élevée s'est rapidement créé, on ne peut décrire les phénomènes dissipatifs par les équations classiques de mouvement de la variable $\vec{r}=\left\{x_{\alpha}\right\}$ avec un terme de friction : $:^{14}$

$$
\mu \ddot{x}_{\alpha}(t)+\sum_{\beta} \gamma_{\alpha \beta}(\vec{r}) \dot{x}_{\beta}(t)+\frac{\partial}{\partial x_{\alpha}} V(\vec{r})=0(10)
$$

oũ le potentiel conservatif est $V(r), \boldsymbol{\gamma}_{\alpha \beta}(r)$ est le coefficient de friction dissipative, et $\alpha=1,2,3$.

La description du flux d'énergie depuis le mouvement collectif vers l'excitation intrinsèque que l'on peut qualifier de "friction" est faite en prenant pour ces modes d'excitation non collectifs le concept d'ensemble canonique statistique. ${ }^{5}$ Même si, en moyenne, ce flux d'énergie est irréversible, les fluctuations thermiques provoquent un couplage entre la variable collective et le bain thermique qui atteint un comportement de hasard ou stochastique. Le caractère de hasard de l'évolution dans le temps de la variable collective apparait. I1 n'est pas possible de prédire exactement la valeur de cette variable à un temps donné. C'est typiquement la propriēté du mouvement Brownien. Dans 1'équation du mouvement dissipatif ${ }^{16} 1$ a force de Langevin ${ }^{17}$ $L_{\alpha}(t)$ intervient; c'est une force dont $1 \mathrm{l}$ valeur moyenne est nulle $<L_{\alpha}(t)>=0$,

$$
\mu \ddot{x}_{\alpha}(t)+\sum_{\beta} \gamma_{\alpha \beta}(\vec{r}) \underset{\beta}{\dot{x}}(t)+\frac{\partial}{\partial x_{\alpha}} V(\vec{r})=L_{\alpha}
$$

Une mêthode proposée depuis très longtemps pour dēcrire les phènomènes de relaxation et de diffusion est connue sous le nom d'équation de Fokker-Planck. Ainsi retourne-t-on au début du 20 e siècle, en jetant un regard ëmu sur les êcrits trop rares de P. Langevin, d'Einstein, de Pauli, ..., dont nous reparlerons dans quelques instants à propos de $1 \mathrm{a}$ "vieille" physique.

Mais avant de terminer avec les domaines nouveaux abordés en physique nucléaire grâce aux ions lourds, citons encore deux thèmes.

Comme tous les objets "mous", les noyaux adaptent leur forme au mouvement de rotation qu'ils subissent . Or pour former un noyau possédant un grand moment angulaire, la méthode évidente est d'apporter avec le projectile un moment angulaire orbital èlevé et de produire un noyau de fusion qui conservera ce spin. Mais cette fusion n'est possible que si la vitesse $n$ 'est pas trop grande. Il faut donc jouer sur la masse.

L'étude de la désexcitation des noyaux de fusion produits par ions lourds consiste entre autre à examiner les rayonnements $\gamma$ correspondant aux transitions de la bande de rotation

$$
E_{\gamma}=\Delta\left(\frac{\ell(\ell+1) \hbar^{2}}{2 y}\right. \text {. }
$$

Comme en spectroscopie moléculaire, on a donc accès au moment d'inertie des noyaux. C'est ainsi qu'avec les très hauts spins, on a constater que les formes devenaient ellipsoïdes, triaxiales, ovoīdes, et que les idées de Bohr et Motteison sur les formes des noyaux en rotation étaient fertiles. Ainsi, la matière nucléaire à l'échelle infiniment petite se comporte-t-elle comme la matière astrale à l'échelle infiniment grande.

L'autre exemple sur lequel je voudrais m'arrêter un peu est 1 'équilibrage en $N / Z$ qui suit très rapidement les collisions dissipatives et la quasifission.

On a observē expērimentalement que les fragments présentaient un rapport neutron-proton non pas voisin soit du projectile, soit de la cible, mais au contraire le rapport du noyau composé correspondant à $\left(A_{1}+A_{2}, Z_{1}+Z_{2}\right)$ ou plus exactement un rapport correspondant à la minimilisation 
de l'énergie potentielle du système. Or, on peut montrer que pour une masse fixēe, $A$, le potentiel est une fonction parabolique de $Z$ (voir figure 8 ). L'équilibration signifierait donc que

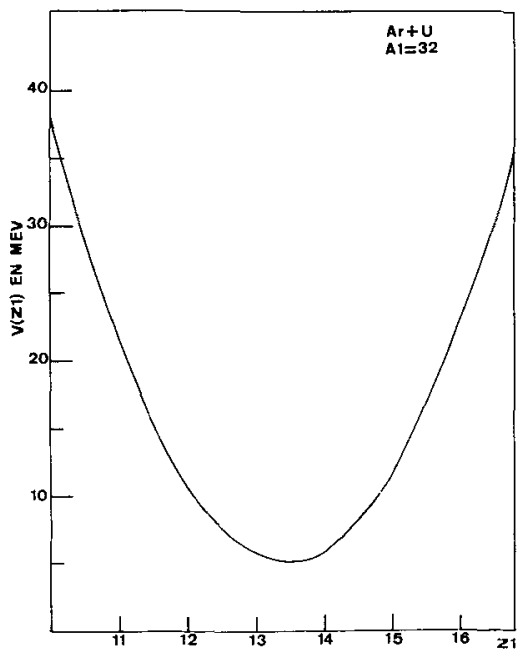

Fig.8. Exemple de potentiel calculé avec deux gouttes liquides jointes par un col pour le systeme $A r+238 U$, pour la masse $A_{1}=32$. C'est dans ce potentiel harmonique que le bain thermique et l'oscillateur quantique s'exercent (Réf. ${ }^{5}$ ).

les interactions conduisent rapidement au minimum du potentiel harmonique. Mais l'on observe une distribution gaussienne autour de cette valeur et la largeur à mi hauteur, ou 1 a variance $\left(\Gamma^{2}=8(\log ) \sigma^{2}\right)$ peuvent être interprétées soit comme un effet de fluctuations statistiques dues à la température (excitation), soit comme un effet quantique, les niveaux d'énergie de phonon dans le potentiel harmonique de la variable $Z$ étant $\hbar \omega\left(n+\frac{1}{2}\right)$. Ceci est liē au problème du mécanisme d'équilibration de $N / Z$ et du premier stade de dissipation d'énergie. Or, lorsque deux noyaux s'interpénétrent, le "mélange" des neutrons n'est pas obligatoirement calqué sur le mélange des protons. L'oscillation du "globe" de neutron par rapport au globe de proton peut être provoquéepar l'absorption de rayonnement électromagnétique de grande énergie. La résonance géante dipolaire a été interprétée de la sorte par Goldhaber et Teller, 11 y a ... 30 ans. On peut imaginer que des oscillations de ce type ont lieu au cours de la collision,et qu'elles provoquent donc une dissipation collective d'énergie conduisant rapidement vers le $N / Z$ le plus stable. Par conséquent, on devrait retrouver l'énergie de phonon dans la mesure de la variance

$$
\sigma^{2}=\frac{1}{K}\left(\frac{\hbar \omega}{2}+\frac{\hbar \omega}{\exp \left(\frac{\hbar \omega}{T}\right)-1}\right)
$$

oũ $k$ est la raideur liêe à la masse effective : $k=m_{\text {eff }} \omega^{2}$ et $T$ la température.

Normalement, l'énergie de phonon $\hbar \omega$, comme d'après la valeur de la résonance géante dans 1 'absorption $\gamma$ devrait être supérieure à $T$, de sorte que l'expression de $\sigma^{2}$ indépendante de $T$ devient

$$
\sigma^{2}=\frac{\hbar \omega}{2 k}
$$

Actuellement, il est encore difficile d'affirmer si les fluctuations sont d'origine statistique ou d'origine quantique. ${ }^{9}$ Le sujet est en pleine discussion mais cette êtude montre bien comment les problèmes sont abordés et comment dans ces noyaux soumis à de fortes perturbations, les idées anciennes -distribution de Boltzmann (1890) et modèle des oscillations pour la résonance isovectorielle $E_{1}$ datant de 1948- sont fertiles pour la nouvelle physique du noyau.

\section{La"vieille physique."}

Et maintenant, continuons de montrer combien la physique nucléaire s'est, à cette occasion, rapprochée du reste de la physique et pourquoi "vieille physique", celle de Boltzmann, d'Einstein, de Rutherford, de Planck, de Pauli, de Chandrasekhar, de Bohr et Wheeler et de quelques autres.

Il suffira de quelques exemples.

Le potentiel de proximité.

Pour représenter quantitativement le potentiel se développant entre deux noyaux qui s'approchent, on a abandonné le modèle optique incapable de décrire les interactions profondes. Le modèle qui décrit le mieux les phénomènes est inspiré de l'étude des forces exercées entre deux surfaces, forces de Van der Vaals, etc...

"La force exercée entre deux surfaces rigides régulièrement courbées est proportionnelle au potentiel par unitê de surface entre surfaces planes, e (s)"

$$
F(s)=2 \pi \frac{R_{1} R_{2}}{R_{1}+R_{2}} \text { e (s) }
$$




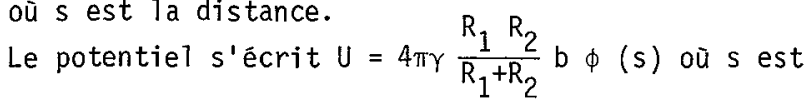
exprimé en fermis et $\gamma$ le coefficient de tension de surface. La fonction $\phi$ est une fonction universelle.

L'idée de ce potentiel, proposée par Blocki et $a 1^{2,0}$ et adopté par tous est neee d'un article de chimie physique vieux de 50 ans, et d'observations et mesures effectuées sur des petites boules de plastique. ${ }^{21}$

Autre exemple encore plus général. Les équations de Fokker-P1anck.

Le moyen le plus général de décrire les phénomènes de relaxation est fourni par 1'équation maitresse de transport de Pauli $i^{2}$ écrite en 1928. Une forme simple date de 1914 . La distribution de probabilité normalisêe $P(x, t)$ pour trouver au temps $t$ le "marcheur au hasard" à la position $x$, obéit à 1 'équation $:{ }^{23}$

$$
\frac{\partial}{\partial t} P(x, t)=-\frac{\partial}{\partial x}\left(v P(x, t)+\frac{1}{2} \frac{\partial^{2}}{\partial x^{2}}\left(D_{x} P(x, t)(16)\right.\right.
$$

Si le coefficient de dérive $v$ et 1 a constante de diffusion $D_{x}$ sont indépendants de $x$ et de $t$, la solution est une gaussienne

$$
P(x, t)=\left(2 \pi D_{x} t\right)^{1 / 2} \exp \left\{-\frac{(x-v t)^{2}}{2 D_{x} t}\right\}
$$

$D_{x}$ décrit 1 'aspect stochastique de la marche.

$v$ indique le sens de la marche.

Le premier moment $\frac{d}{d t}\langle x(t)\rangle=\langle v\rangle$

Le deuxième moment $\frac{d}{d t}\left(\left\langle x^{2}(t)\right\rangle-\langle x(t)\rangle^{2}\right)=$ $\left\langle D_{x}\right\rangle+2(\langle x v\rangle-\langle x\rangle\langle v\rangle)(19)$

Ce type d'équation a été utilisé pour décrire l'évolution des variables suivantes: énergie cinétique du mouvement relatif, $P_{\text {rad }}$, transfert de moment angulaire, $p_{\theta}$, asymétrie de masse $\frac{A_{2}-A_{1}}{A_{1}+A_{2}}$, et même $N / Z$. Les rêsultats expérimentaux correspondaient aux sections efficaces différentielles $\frac{d^{2} \sigma}{d E d \theta}, \frac{d^{2} \sigma}{d A d \theta}, \frac{d^{3} \sigma}{d A d E d \theta}$, etc. .

C'est en 1905 qu'Einstein, dans son analyse du mouvement Brownien, a montré que $v$ et $D$ sont liēs par la température du bain $\frac{v}{D}=\frac{C}{2 k T}$, k étant la constante de Boltzmann.

On a pu montrer ${ }^{2}{ }^{5}$ comparant les coefficients déduits des expériences à différentes énergies d'excitation - c'est-à-dire, différentes pertes d'ênergies cinétiques, que la relation d'Einstein s'applique bien aux noyaux en interaction (voir figure 9).

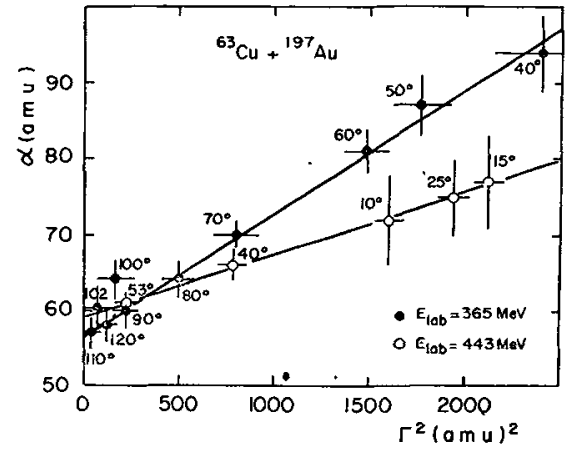

Fig.9. Illustration de la relation d'Einstein entre le coefficient de dérive la est la position du maximum de la distribution de masse) et le coefficient de diffusion ( $\Gamma$ est $l_{a}$. Zargeur à mi hauteur de la distribution) (équation (20) dans $i_{1}$ texte). Le popport $\alpha / T^{2}$ est égat $\grave{a} \frac{M_{1}}{(8 \log 2) M_{2}}=\frac{C}{(16 \log 2) T}$ et ne dépend pas de l'angle d'observation $\theta$, dono du temps. Les deux droites correspondent à deux énergies d'excitation, done d deux températures (Réf. ${ }^{25}$ ).

Le modèle de la boule de feútécrivant les réactions périphêriques à très haute ênergie est une autre illustration. Le projectile traverse la cible et sa température s'élève au point d'atteindre une valeur qui pourrait être le point d'ébullition de la matière nucléaire. Selon Scott', à partir d'une certaine énergie, la température sature à environ $8 \mathrm{MeV}$, valeur prévisible en considérant le gaz de Fermi du noyau (voir figure 10).

Il faudrait citer bien d'autres exemples : la dissipation à 1 corps (les nucléons frappent la surface d'un piston qui représente le potentiel nuclēaire dans son ensemble, mais n'êchangent pas d'énergie dans des collisions entre éux), les vibrations de surface des noyaux et les résonances ${ }^{27}$ qui en résultent, directement inspirées des vibrations des molécules, 1'usage extensif des fonctions de dēflexion qui était bien tombé de mode depuis Rutherford. ${ }^{28}$

Comme toutes les nouveautés, la physique nucléaire macroscopique des ions lourds devient au cours du temps de moins en moins nouvelle. Plus de dix ans déjà... La vieillesse arrivera d'autant 


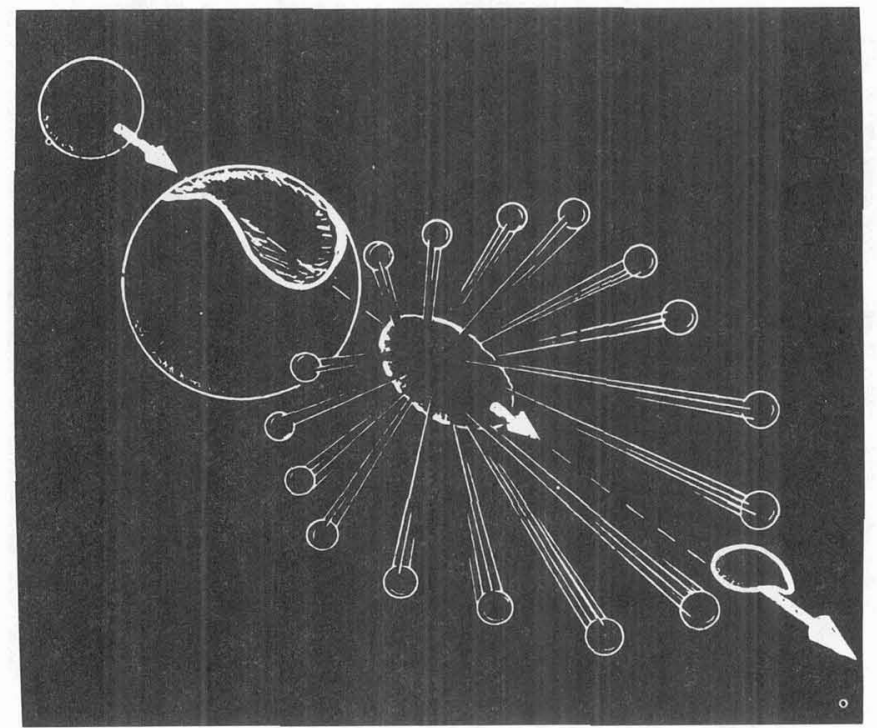

Fig.10. La boule de feu. Illustration $\bar{d}^{\prime}$ une interaction périphérique à très haute énergie d'après Gutbrod et Pskanger (Réf. ${ }^{26}$ ).

plus tard que la routine sera évitêe. Après une période oũ chaque expérience apportait une information inattendue, une analyse plus en profondeur bien que moins spectaculaire est nécessaire et se développe. Elle est encore limitée par le manque d'accélérateurs dans la zone de 10 à $200 \mathrm{MeV}$ par nucléon. L'avenir est sans doute dans une synthèse entre les descriptions macroscopiques et microscopiques, statistiques et quantiques, entre l'étude dynamique des collisions et les aspects de structure des noyaux.

Pour terminer, permettez moi de revenir au début : le propos de Roger Caillois ne porte que sur les apparences. Un découverte chasse l'autre, i1 est vrai, mais les connaissances un moment mises à 1 'ombre reviennent. Je continue de croire à la persévérance. Après tout, personne ne croyait, sauf l'auteur, à l'article du Journal de Physique de 1960 sur les réactions par ions lourds ${ }^{29}$. Cependant, en 1966, le cyclotron d'Orsay CEVIL dêlivrait les premiers faisceaux en France d'Azote, d'0xygène et de Carbone. La critique était encore vive aux Journées de La Plagne organisêes ${ }^{30}$ par I'I.P.N. de Lyon en 1969, mais en 1969, les réac- tions dissipatives étaient découvertes ${ }^{31}$ et en 1970, ALICE fournissait le premier faisceau mondial de Krypton, grâce auquel la quasi-fission était trouvée ${ }^{32}$.

Comme le ditt3 le poète Latin "Ipse seram teneras maturo tempore vites": "Je planterai de mes mains des tendres vignes à 7 'époque voulue".

Espérons qu'une récolte trop abondante n'épuisera pas le cep.

\section{REFERENCEES}

Roger Caillois. Le Nouvel Observateur. Nov. 1978.

2 C. Bieth, M. Gouttefangeas, S. Harar, M. Lefort GANIL. Proc. Int. Conf. Nuclear Structure Tokyo. J. Phys. Soc. Japan 44, 589 (1978).

3 J. Arianer et a], Cryebis. C.R. Acad. Sciences 288. B 111 (1979).

4 R. Geller (Electron cyclotron resonance source) 8 th Conference on cyclotrons.I.E.E.E. Trans. Nucl. Sci. (April 1979).

5 T.H. Chiang, D. Guerreau, P. Auger, J. Galin, B. Gatty, X. Tarrago, J. Girard. IPNO-RC-79.01 (1979).

6 L.S. Schroeder. Proc. Topical Meeting on multiparticle production of nuclei at very high energy. ICTP Trieste (1976).

7 R. Bock, A. Gobbi, H. Sann, H. Stelzer, U. Lynen, A. OTmi Sann GSI 79.3 (1979).

\& S. Della Negra, C. Deprun, H. Jungclass, $H$. Gauvin, J.P. Husson, Y. Le Beyec. Nucl. Instr. Methods. 156, 355 (1978).

9 H.J. Specht et Phys. Rev. Lett. in press (1979)

10 M. Lefort, C. Ngô. Annales Physique 3, 5-85 (1978).

11 M. Lefort. Erice School on Nuclear Physics. April (1979). Progress in Nuclear Physics. Pergamon Press.

12 D.K. Scott. LBL 7727 (1978).

13 J. Wilczynski. Phys. Lett. 47B, 484 (1973).

14 D.H.E. Gross, H. Kalinowski. Phys. Lett. 48B, 302 (1974).

15 W. Nörenberg. Phys. Lett. 52B, 289 (1974).

16 H.A. WeidenmütTer. Journal Phys. Soc. Japan. 44, supp1. p.701 (1978).

17 P. Langevin. C.R. Acad. Sciences 146, 530 (1908)

18 M. Goldhaber, E. Teller. Phys. Rev. 74, 1046, (1948).

19 M. Berlanger, A. Gobbi, F. Hanappe, U. Lynen, C. Ngô, A. Olmi, H. Sann, H. Stelzer, M.F. Rivet. Zeit. Phys. A. 291, 133 (1979).

20 J. Blocki, J. Randrup, W.J. Swiatecki, C.F. Tsang. Ann. Phys. 105, 427 (1977). 
21 K.L. Johnson, K. Kenda11, A.D. Roberts, Proc. Roy. Soc. 324, 301 (1971).

22 W. Pauli. Festschrift zum 60. Geburtstag A. Sommerfelds $P$. Debye éditeur p.30 HirzeTVerlag Leipzig (1928).

23 S. Chandrasekhar. Rev. Mod. Phys. 15, 1(1943) A.D. Fokker. Ann.d.Physik. 43, 812 (1914) M. Planck.Sitz der Preuss Akad. 324 (1917)

24 A. Einstein. Ann. Phys. 17, 549 (1905)

25 C. Ngô, J. Peter, B. Tamain, M. Berlanger, F. Hanappe, Nucl. Phys. A267, 181 (1976)

26 J. Gosset, H.H. Gutbrod, W.G. Meyer, A.M. Poskanzer, A. Sandoval, R. Stock, G.D. Westfall. Phys. Rev. C.16, 629 (1977).

27 R.A. Broglia, C.H. Dasso, G. Pollarolo, A. Winther. Phys. Rev. Lett. 41, 25 (1978)

28 Lord Rutherford. Phil Mag 669 (1911).

29 M. Lefort. J. Physique 21, 666 (1960).

30 le Session d'études biennales de Physique Nucléaire de T'I.P.N. de Lyon. Lycen 69.01 La Plagne (1969).

31 J. Galin, B. Gatty, M. Lefort, J. Peter, X. Tarrago, R. Basile. Phys. Rev. 182, 1267(1969)

32 M. Lefort, C. Ngô, J. Peter, B. Tamain. Nucl. Phys. A216, 166 (1973).

з3 Tibulle. Elegies. 